\title{
Low-Cost, Precision, Self-Alignment Technique for Coupling Laser and Photodiode Arrays to Polymer Waveguide Arrays on Multilayer PCBs
}

\author{
Ioannis Papakonstantinou, Student Member, IEEE, David R. Selviah, Member, IEEE, Richard C. A. Pitwon, and
} Dave Milward

\begin{abstract}
The first, to our knowledge, passive, precision, selfalignment technique for direct coupling of vertical cavity surface emitting laser (VCSEL) and photodiode (PD) arrays to an array of polymer buried channel waveguides on a rigid printed circuit board (PCB) is reported. It gives insertion losses as good as the best achieved previously, to within experimental measurement accuracy, but without the need for costly active alignment nor waveguide facet polishing and so is a major step towards a commercially realizable low cost connector. Such an optical connector with four duplex channels each operating at $10 \mathrm{~Gb} / \mathrm{s}(80 \mathrm{~Gb} / \mathrm{s}$ aggregate) was designed, constructed, and its alignment precision assessed. The alignment technique is applicable to polymer waveguide interconnections on both rigid and flexible multilayer printed circuit boards (PCBs). The dependence of optical coupling loss on misalignments in $x, y$ and $z$ of the VCSEL and PD arrays allows the precision of alignment to be assessed and its reproducibility on multiple mating cycles of the connector is reported. The first recorded measurements of crosstalk between waveguides when the connector is misaligned are reported. Lateral misalignments of the connector to within its tolerance are shown to have no effect on the signal to crosstalk ratio (SCR), to within experimental measurement accuracy. The insertion loss repeatability is similar to that of single mode fiber mechanically transferable (MT) connectors.
\end{abstract}

Index Terms-Alignment tolerances, optical connectors, optical interconnections, optical waveguide crosstalk, polymer waveguides.

\section{INTRODUCTION}

$\mathbf{O}$ PTICAL interconnections are being investigated for short distance data communication applications on printed circuit boards (PCBs) to replace copper tracks which suffer severe crosstalk, increased loss, and increased fabrication cost as data rates rise above $10 \mathrm{~Gb} / \mathrm{s}$. Optical beams can pass through

Manuscript received September 5, 2006; revised July 18, 2007. First published May 30, 2008; last published August 6, 2008 (projected). This work was supported by the U.K. Department of Trade and Industry (DTI) and the U.K. Engineering and Physical Sciences Research Council (EPSRC), via LINK Information Storage and Displays "Storlite" grant GR/S28136/01 and via EPSRC DTA. The work of I. Papakonstantinou was supported by Xyratex Technology Ltd.. This work was recommended for publication by Associate Editor E. Suhir upon evaluation of the reviewers comments.

I. Papakonstantinou and D. R. Selviah are with the Department of Electronic and Electrical Engineering, University College, London, London WC1E 7JE U.K. (e-mail: d.selviah@ee.ucl.ac.uk; i.papakonstantinou@ee.ucl.ac.uk).

R. C. A. Pitwon and D. Milward are with Xyratex Technology Ltd., Hampshire PO9 1SA, U.K. (e-mail: dave_milward@xyratex.com; richard_pitwon@xyratex.com).

Color versions of one or more of the figures in this paper are available online at http://ieeexplore.ieee.org.

Digital Object Identifier 10.1109/TADVP.2008.924243 one another in vacuo without any crosstalk and so offer an attractive alternative provided the cost of the optical interconnections are competitive. The lowest cost approach is to form polymer waveguides [1]-[3] on multilayer PCBs which has the advantage that the PCB copper tracks can carry power and low data rate control signals to mezzanine boards while the polymer waveguides carry high data rate optical signals. The cost of the connector is the limiting factor, which increases if higher alignment tolerances are required. Therefore, to minimize this cost single mode waveguides are avoided and larger diameter multimode waveguides are preferred to give larger lateral, in-plane, $x$, and transverse, normal to the plane, $y$, alignment tolerances.

The most popular connector designs [4]-[12] use a vertical cavity surface emitting laser (VCSEL) emitting normally, into the planar surface of the waveguide layer and a $45^{\circ}$ angled plane mirror reflects the light through $90^{\circ}$ into the waveguide. Although $45^{\circ}$ mirror fabrication techniques have given losses as low as $0.5-1.5 \mathrm{~dB}$ per mirror [4]-[12] they are still being optimized to reduce the fabrication cost and to reproducibly give the correct angle and surface flatness over large optical PCB (OPCB) areas.

A more direct technique in which a VCSEL is butt-coupled to the end of the waveguide [14]-[16] gives lower loss due to the absence of the mirror but is difficult to align and can only be used at the edges or in holes in the OPCB. $5 \mathrm{~Gb} / \mathrm{s}$ [16] transmission has been demonstrated but it was limited by losses at the connector interfaces. So the major challenge is to avoid the need for costly and time consuming active alignment [13] to maximize light throughput by designing a low-cost, self-aligning connector which may be attached and detached repeatedly being robust enough to give a consistent coupling loss.

Mechanically transferable (MT) plugs and sockets have been used by several research groups [5], [6], [14], [15] as a basis for making connectors as they are easily fabricated and have two long registration pins and guide holes which give good translational and angular alignment of the plug to the socket. VCSELs can easily and accurately be cleaved singly or as arrays along cleaving lines and located against raised alignment features or projections in the plug. In this way, their alignment with respect to the MT plug pins is determined by the alignment precision of the pins to the alignment features which can be achieved to within $1 \mu \mathrm{m}$ [17], [23]. However, the most serious problem is in the alignment of the MT-socket to the waveguides and this has prevented a practical, low-cost, detachable connector being brought to the market until now. 
Section II describes our low-cost, passive, self-alignment technique. Section III describes the structure of a prototype multiple channel connector. Section IV measures the optical coupling loss with respect to mechanical misalignments of the laser and the photodetector (PD), the crosstalk between neighboring waveguides, and the reproducibility of coupling loss on multiple connector mating cycles. Section V presents the conclusion.

\section{DeVelopment of A TeChNiQue For Self-Alignment OF THE MT-SOCKET TO THE WAVEGUIDES}

\section{A. Difficulties of Alignment}

Low cost PCBs are commonly made from FR4 which is a nonuniform woven material having various temperature expansion coefficients in different directions [18], while its thickness may vary significantly from sample to sample [19]. So, if the MT-socket is attached to the bottom of the FR4 substrate first it will move with respect to the top of the substrate where the waveguides are formed resulting in misalignment. If the socket is similarly attached to different FR4 substrates misalignment with respect to the waveguides on the upper surface occurs due to sample thickness variability. In addition, the woven pattern of the FR4 often results in a rippling of its surface [19]. As a result, if the MT-socket is attached to the top of the FR4 substrate there will be a large uncertainty in the alignment to the waveguide cores. FR4 substrate wafers can be planarized by depositing a sufficiently thick layer of lower waveguide cladding polymer, but there is still a large uncertainty in its final thickness [20]. Therefore, the connector socket cannot be attached only to the lower surface of the PCB. A practical precision alignment technique must be made independent of the thickness of either the FR4 substrate and of the thickness of the polymer waveguide lower cladding.

One popular photosensitive acrylate polymer for fabricating waveguides is Truemode which has a low loss at 850 -nm wavelength and is robust to humidity and thermal cycling but it must be used wet after spinning it onto the substrate [21]. In the case of lithographic fabrication, the mask must be offset by about $100 \mu \mathrm{m}$ above the surface of the wet polymer to avoid adhesion effects and this spacing may vary from one fabricated batch to another [20]. The offset may cause a less well-defined waveguide with walls sloping outwards towards its base and overexposure may give wider waveguides than those defined on the dark field mask. If the MT-socket is laterally aligned in the PCB plane to visual alignment features such as electrical vias or exposed copper tracks or specifically formed fiducial marks on the FR4 surface then for the socket to align to the waveguides the waveguides must be fabricated so that they align to the PCB visual alignment features. However, the mask required is a dark field mask so it is difficult to see alignment features below the clear wet polymer layer. Moreover, since the mask is $100 \mu \mathrm{m}$ plus the thickness of the core and lower cladding above the FR4 it is difficult to bring the visual alignment features on the FR4 and the waveguides on the mask into focus together resulting in very poor lateral alignment. Tighter process control and calibrated dual focus mask aligners can improve reproducibility of

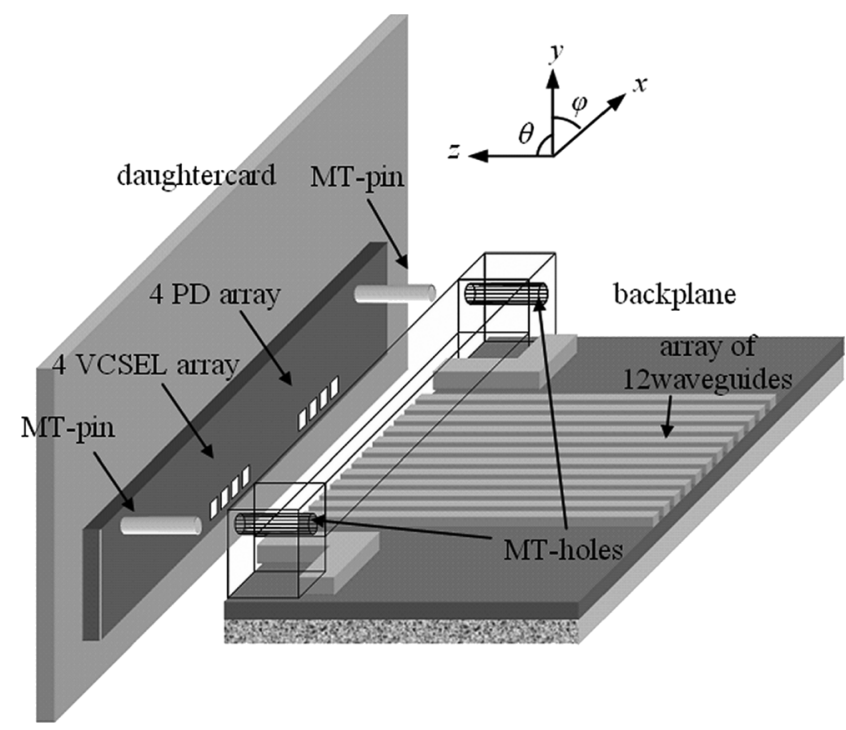

Fig. 1. Alignment method based on MT concept. After registering the MT-pins of the plug into the MT-holes of the socket accurate alignment between the VCSEL's-PIN diodes of the mezzanine board and the waveguides of the backplane is achieved.

results but at an increased cost penalty to the fabrication of the complete structure.

\section{B. Practical Self-Alignment Technique}

We propose the use of an MT-socket interposer (Fig. 1), which is micromechanically aligned to the waveguides. The MT-socket interposer (Fig. 2) is an inverted U-shaped polymer structure that fits over the linear array of waveguides. The first key aspect of the alignment technique is that the 2 feet of the MT-socket interposer stand on the top of the lower cladding layer, which acts as the datum layer with respect to which the alignment is achieved. As the waveguides are also formed on the same datum layer, the MT-socket interposer maintains the same $y$ alignment with the waveguides irrespective of any lower layers.

The second key aspect of the alignment technique is that the mask used to fabricate the waveguides is also used to fabricate mechanical alignment features at the same time in the core layer having the same thickness as the waveguides with sidewalls running parallel to the waveguides. This ensures correct lateral positioning of the alignment features due to the high positional accuracy of the mask layout. In our work, a high precision e-beam mask was used to expose the core layer. The spacing between the inner faces of the MT-socket interposer legs is designed to match the spacing between the outer sidewalls of the mechanical alignment features formed in the core layer (Fig. 2). So when the MT-socket interposer is placed by hand over the alignment features and moved laterally in $x$ it easily slots down into place on the datum level of the lower cladding. In this way the MT-socket, interposer mechanically self-aligns.

The alignment in $z$ is achieved by forming the mechanical alignment features to have backstops so that once the MT-socket interposer has been slotted into place laterally it can simply be slid back into contact with the backstops. Finally, 


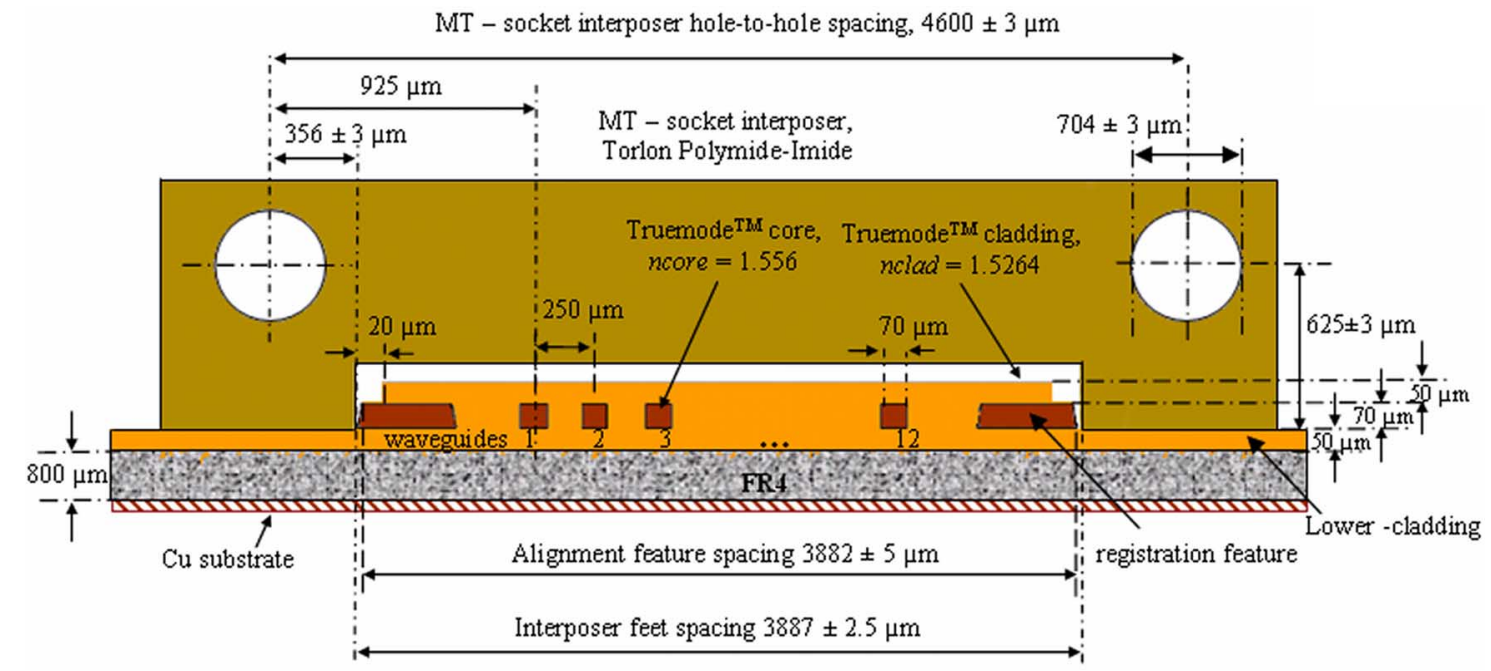

Fig. 2. MT-socket interposer slotted into place on the top of the lower cladding in contact with the exposed alignment feature walls with dimensions. Diagram is not in scale to allow important dimensions to be clearly displayed. Critical for the operation of the connector dimensions are given with their tolerances.

while clamping the MT-socket interposer in place glue can be put around its legs to lock it into place.

The third key aspect is that when the waveguides are covered with the upper cladding to bury them it is arranged so that it does not cover the aligning side wall nor back stop wall of the mechanical alignment features so that these remain exposed and available (Fig. 1) for mechanical contact alignment of the feet of the MT-socket interposer. Provided the upper cladding is sufficiently stepped back from the alignment wall in the core layer then the lateral tolerance for positioning the upper cladding layer need not be very tight. This is easily achieved using a photomask with which to expose the upper cladding layer, as the highest precision of e-beam masks is not required. The upper cladding mask is light field and so it is easy to see and align to alignment marks in the core layer. In our work, two cross-shaped visual alignment marks were formed in the core layer for this purpose (Fig. 4).

The sidewalls sometimes slope outwards towards the base of the waveguide (Fig. 2). The waveguides are sometimes overexposed giving waveguides and alignment features that are larger than on the mask by a certain amount and this needs to be taken into account in designing the spacing of the 2 feet of the MT-socket interposer as the feet will make contact with the bottom of the waveguide walls. Generally, the lateral positions of the sidewalls of the mechanical alignment features are reproducible but if the process control is insufficient, there may be an uncertainty in the lateral position of the sidewalls. In this case, the MT-socket interposer feet can be shaped so that they have a wider spacing away from the cut edge of the waveguides. Similarly, the alignment features can be angled so that they have a wider spacing away from the cut edge of the waveguides. Alignment is then achieved by placing the MT-socket interposer onto the lower cladding layer and then sliding it back until it is in contact with the mechanical alignment features. This ensures perfect lateral alignment irrespective of over or under exposure through the mask; however, it is at the expense of some increased uncertainty in the axial, $z$ position of the socket. However, as we show later a large amount of misalignment in $z$, is possible without serious ill effects on the coupling loss whereas accurate lateral alignment is far more important.

An interesting technique to align waveguide arrays to 2-D arrays of VCSELs and PDs using MT ferrules was reported in [6]. In that approach, waveguides were formed in a grid pattern on several glass wafers, which were aligned above each other with the aid of visual alignment marks and subsequently laminated. The resulting wafer-stack was then diced across the cores to form a 3-D waveguide array with $45^{\circ}$ optical path redirection mirrors. The final step was to dice the wafer-stack into rectangular blocks that could fit into the pit of an MT ferrule. A 50- $\mu \mathrm{m}$ gap was left between either side of the waveguide block and the MT pit probably to allow for inaccuracies involved in the dicing process which is difficult to control to high precision. As a result active lateral alignment had to be used to align the waveguides and the VCSEL and PD arrays along the $x$-direction. However, vertical alignment along $y$ was passive, since the glass wafer thicknesses could be controlled to very high accuracy. These last two features significantly differentiate the work reported in [6] from our work presented here. First, in our work the thickness of the polymer lower cladding, core and upper cladding layers is controlled by the spin coating fabrication step giving a maximum of $10 \%$ variation in the thickness of each layer. So alignment in $y$ cannot rely on the polymer layer thicknesses. This problem is overcome in our technique by resting the feet of the MT-socket interposer on the top of the lower-cladding layer as shown in Fig. 2. In this way, the vertical MT-hole to waveguide alignment $y$ depends only on the distance from the bottom of the interposer feet to the center of the MT-holes, which can be very precisely controlled. Second, the lateral alignment $x$ depends on the lateral position of the alignment features Fig. 2, with respect to the waveguides. Since both are formed simultaneously and with the aid of the same e-beam mask, accurate alignment in $x$ is guaranteed. In conclusion, alignment in our connector was achieved completely passively along the two critical $x$ and $y$-directions and along the $z$-direction without any costly and time-consuming active step being involved. 
(a)

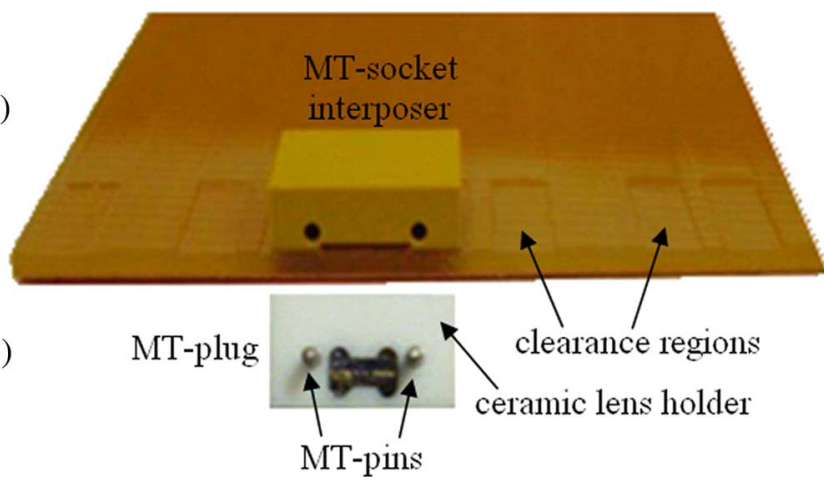

Fig. 3. (a) Optoelectronic PCB with MT-socket interposer mounted on the surface about to slide back into position against backstops. (b) MT-plug with ceramic lens holder in place.

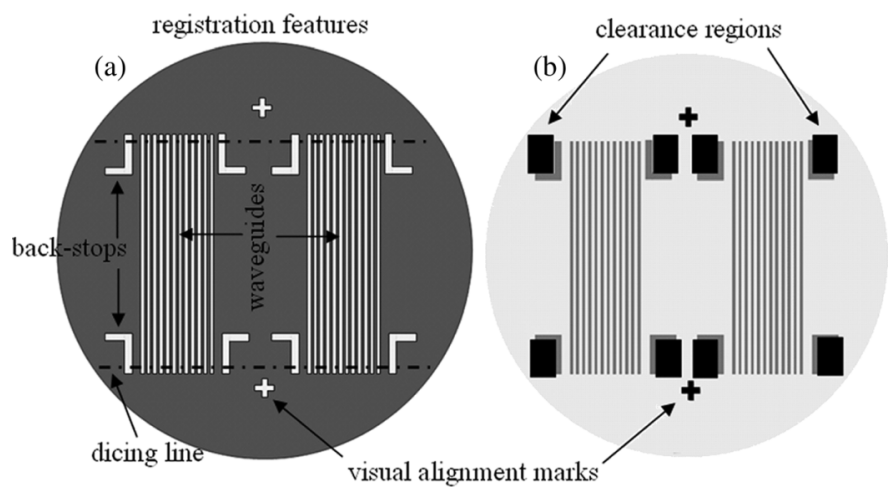

Fig. 4. (a) Core layer mask. Waveguides, mechanical registration features, mechanical backstops, and optical alignment marks for the cladding-mask are shown. (b) Upper-cladding mask as it appears in the mask aligner on top of an incompletely processed wafer. Features of cladding mask are opaque and shown in black while core features on the wafer are shown in gray.

Finally, we note that our alignment technique is suitable for use on both rigid and flexible multilayer PCBs as the MT-socket interposer is only glued over a small area of the $\mathrm{PCB}$. The technique can also be extended for use with multiple layers of waveguides by using 2-D arrays of VCSELs, PDs, and lenses in the MT-plug.

\section{PRototype CONNECTOR DESIGN AND CONSTRUCTION}

A prototype connector was designed making use of the novel micromechanical alignment technique. This enabled the degree of alignment of this technique to be assessed as well as the effect of the misalignment on the optical coupling, insertion loss, and crosstalk. It is desirable to have no active devices, which may fail, on the optical PCB backplane, as this is usually an intimate part of the rack-based system and difficult to remove for repair. Therefore, all of the active devices were mounted inside the MT-plug of the connector and the MT-socket had no active devices. The connector was designed to have four duplex channels or eight singleton channels each capable of operating at $10 \mathrm{~Gb} / \mathrm{s}$. The connector contained a linear array of four laser sources and four photodiode detectors and the MT-socket interposer was placed over 12 waveguides although in this demonstration only the four at each end were used.

\section{A. MT-Plug}

Low cost 850-nm wavelength $10 \mathrm{~Gb} / \mathrm{s}$ VCSELs with $7 \mu \mathrm{m}$ diameter optical apertures were chosen as the optical sources. $10 \mathrm{~Gb} / \mathrm{s}$ photodetectors (PDs) with the largest optical apertures available, GaAs PIN PDs with $75 \mu \mathrm{m}$ diameters were selected to so that tight alignment tolerances were not required. Linear arrays of 4 VCSELs and of four photodiodes on a pitch of $250 \mu \mathrm{m}$ to match that of the waveguides were aligned and glued onto the MT-plug header using its raised alignment features and were wire bonded to electrical contacts [Fig. 3(b)]. The VCSELs were arranged to launch light into waveguides 1-4 and the PDs to receive light from waveguides 9-12. Waveguides 5-8 were unused in this demonstration. To avoid the bond wires being knocked off when butt-coupled to the waveguides we adopted a novel approach of using an array of imaging GRIN lenses to image the VCSELs and PDs to a position on the GRIN lens face nearest the waveguides. The GRIN lenses were designed to allow them to be positioned beyond the bond wires of the VCSEL so that they were not affected. Apart from protecting the bond wires, the GRIN lenses could be physically butt coupled to the waveguides with some degree of force without damaging the VCSELs if they had been left exposed.

The GRIN lenses were mounted on silicon V-grooves in a ceramic holder [Fig. 3(b)]. The ceramic lens holder had two $705 \pm 3 \mu \mathrm{m}$ diameter holes corresponding but having a slightly larger diameter than that of the MT-plug pins. This was done so that it could slip over them and then be adjusted in $x$, and $y$ under a microscope to bring the image of the VCSELs and PDs into focus at the center of the front surface of the GRIN lenses. Subsequent measurement of the assembly showed that in fact the active devices were imaged $2 \mu \mathrm{m}$ beyond the face of the GRIN lenses towards the waveguides. In a commercial product the tolerances would be tightened so that no active alignment is required in the plug.

\section{B. MT-Socket, Waveguides, and Alignment Features}

The MT-socket interposer, Fig. 2 and Fig. 3(a) for the prototype was machined from a block of Torlon Polyamide-imide (PAI) which has a thermal expansion coefficient of $14.4 \mathrm{ppm} /{ }^{\circ} \mathrm{C}$ chosen to be compatible with that of the FR4. The interposer was designed to be injection molded to minimize cost in a final product.

Each waveguide was designed to have a square cross section of $70 \mu \mathrm{m} \times 70 \mu \mathrm{m}$ to maximize the misalignment possible while minimizing the coupling loss and the crosstalk between adjacent waveguides for our choice of VCSEL and PD diameters. The array of 12 straight waveguides were on a pitch of $250 \mu \mathrm{m}$ and were $10 \mathrm{~cm}$ long to fit onto a 7-in diameter circular FR4 wafer substrate of $800 \mu \mathrm{m}$ thickness coated in a copper layer of $17 \mu \mathrm{m}$ thickness on the opposite PCB surface from that on which the waveguides were formed. Following spinning and UV curing of the $\sim 50-\mu \mathrm{m}$-thick lower cladding planarizing layer the core layer was spun on to a thickness of $70 \mu \mathrm{m}$. Waveguides and alignment features with backstops were formed photolithographically in the core layer using an electron-beam dark field mask [Fig. 4(a)]. After UV exposure through the mask and removal of the unexposed core material an upper cladding polymer layer was spun over the whole substrate to a depth of 
TABLE I

TOLERANCE Along $X, Y$ AND, $Z$ FOR CONNECTOR COMPONENTS

\begin{tabular}{|c|c|c|c|}
\hline & $X$ & $y$ & $Z$ \\
\hline MT-plug & $\pm 3 \mu \mathrm{m}$ (pin-to-pin) & $\pm 3 \mu \mathrm{m}$ (pin-to-GRIN) & $\longrightarrow$ \\
\hline MT-socket & $\pm 3 \mu \mathrm{m}$ (hole-to-hole) & $\pm 3 \mu \mathrm{m}$ (hole-to-waveguide) & $\longrightarrow$ \\
\hline OPCB features & $\begin{array}{l}+2.5 \mu \mathrm{m} \text { (increase in registration wall-to-wall } \\
\text { spacing due to overexposure) } \\
\pm 2.5 \mu \mathrm{m} \text { (due to } 5 \mu \mathrm{m} \text { extra spacing between } \\
\text { feet of interposer) }\end{array}$ & $\pm 1 \mu \mathrm{m}$ (core thickness control) & $\begin{array}{c}+10 \mu \mathrm{m} \text { (accuracy of dicing in respect to } \\
\text { the dicing lines on the board) } \\
+2.5 \mu \mathrm{m} \text { (backstop shift due to } \\
\text { overetching) }\end{array}$ \\
\hline $\begin{array}{c}\text { Tolerance of MT } \\
\text { interposer socket to } \\
\text { waveguides }\end{array}$ & $\begin{array}{c} \pm 8 \mu \mathrm{m} \text { or } \pm 3 \mu \mathrm{m} \text { if overexposure widening is } \\
\text { known and reproducible }\end{array}$ & $\pm 4 \mu \mathrm{m}$ & $\begin{array}{c}+12.5 \mu \mathrm{m} \text { or }+10 \mu \mathrm{m} \text { if overexposure } \\
\text { widening is known and reproducible }\end{array}$ \\
\hline $\begin{array}{l}\text { Combined tolerance of } \\
\text { VCSEL and PIN to } \\
\text { waveguides }\end{array}$ & $\begin{array}{l} \pm 11 \mu \mathrm{m} \text { or } \pm 6 \mu \mathrm{m} \text { if overexposure widening is } \\
\text { known and reproducible }\end{array}$ & $\pm 7 \mu \mathrm{m}$ & $\begin{array}{l}+12.5 \mu \mathrm{m} \text { or }+10 \mu \mathrm{m} \text { if overexposure } \\
\text { widening is known and reproducible }\end{array}$ \\
\hline
\end{tabular}

$\sim 50 \mu \mathrm{m}$ above the top of the waveguides. A second light field photomask [Fig. 4(b)] was aligned to the visual cross alignment features left in the core layer from the first mask [Fig. 4(a)]. Critical visual alignment was not necessary since a gap of $20 \mu \mathrm{m}$ had been left [Fig. 2] between the edge of the upper cladding and the exposed core alignment feature walls. This also meant that a lower cost lower resolution photomask was sufficient. The upper cladding was exposed by UV illumination through the second mask and the unexposed upper cladding material washed off. The upper cladding polymer was the same as the lower cladding polymer and had the same lower refractive index than the core when cured. The refractive index of the core after UV exposure was $n_{\text {core }}=1.556$ and that of the cladding was $n_{\text {clad }}=1.5264$, as measured by the prism coupler method, giving a numerical aperture of $N A_{\text {waveguide }}=0.302$.

The separation of the internal faces of the MT-interposer feet was designed to be $5 \mu \mathrm{m}$ wider than the spacing between the outer walls of the alignment features on the mask to allow for the expected overexposure and wall slope which caused widening of the alignment feature wall spacing.

\section{Connector Alignment Tolerances}

Translational misalignments can occur in the horizontal direction, in plane $x$ in the vertical direction, normal to the plane $y$ in the axial direction $z$ and angular misalignments can occur in $\theta$ and $\varphi$. The amounts of misalignment are determined by the fabrication accuracies of each of the MT-plug, MT-socket, and OPCB features. The individual and combined tolerances are summarized in Table I for along all three translational directions. The tolerances for the MT-plug and MT-socket were obtained from the manufacturers while those for the OPCB features were measured by shadowgraph (Fig. 5) in which highly collimated white light is passed through the MT-socket and waveguide assembly and cast a shadow on a screen, which is then magnified. The shadowgraph clearly shows the waveguides, alignment features, and the MT-socket interposer and their relative alignments in $x$ and $y$, to scale. The measured separation of the alignment feature walls was $3886 \mu \mathrm{m}$, which is larger than the separation designed on the mask of $3882 \mu \mathrm{m}$ as expected due to over exposure and wall slope. The worst case combined misalignment of VCSEL and PIN to waveguides in Table I along $x$ is $\pm 11 \mu \mathrm{m}$, along $y$ is $\pm 7 \mu \mathrm{m}$, and along $z$ is $+12.5 \mu \mathrm{m}$. However, in a well-characterized fabrication, process the overexposure would be known and reproducible

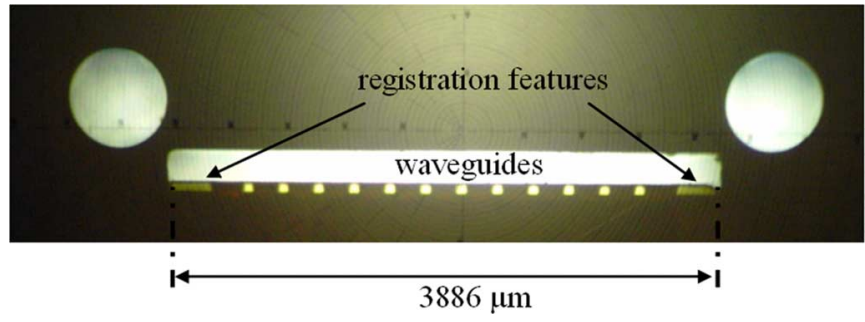

Fig. 5. Shadowgraph picture showing the actual alignment of the components.

and so the alignment error in $x$ reduces to $\pm 6 \mu \mathrm{m}$ and in $z$ to $+10 \mu \mathrm{m}$. The worst angular error in standard MT connectors is $\theta$ and $\varphi$ of $0.01^{\circ}$, which is expected to have negligible effect on insertion loss [23].

\section{EXPERIMENTAL MEASUREMENTS}

\section{A. Misaligned Insertion Loss and Waveguide Selection}

The choice of $70-\mu \mathrm{m}$ square cross-section waveguides was based on evidence from preliminary measurements (Fig. 6), of the power coupled into a test sample array of 15 waveguides of various widths. The thickness of these waveguides was $50 \mu \mathrm{m}$, constant throughout the array, while their widths ranged from 10 to $150 \mu \mathrm{m}$ with $10 \mu \mathrm{m}$ difference between successive waveguides. The lateral separation between two adjacent waveguides centers was $250 \mu \mathrm{m}$. In our experiments, we positioned an MT-plug, after its pins had been removed, on a system of three motorized $x, y, z$ stages and we brought the GRIN-lenses in front of the VCSELs into contact with one of the waveguides on the wafer. The exit of this same waveguide was then butt-coupled to a $75-\mu \mathrm{m}$ pinhole attached via a GRIN lens to a GaAs photodetector, to simulate a $75-\mu \mathrm{m}$ aperture PD imaged through the GRIN lens. The MT-plug and pinhole were each adjusted along the two lateral directions until the power received by the PD was maximized.

Once the insertion loss of that waveguide had been measured, we moved the MT-plug holding the VCSEL and GRIN lens array, first by $250 \mu \mathrm{m}$ to the left and then by $250 \mu \mathrm{m}$ to the right of this waveguide without moving the pinhole and photodiode from its original position. The power recorded on the photodiode represented the crosstalk, which is different on each side of the central waveguide due to the asymmetry in their widths. In this way, we measured the signal to crosstalk ratio (SCR) that 


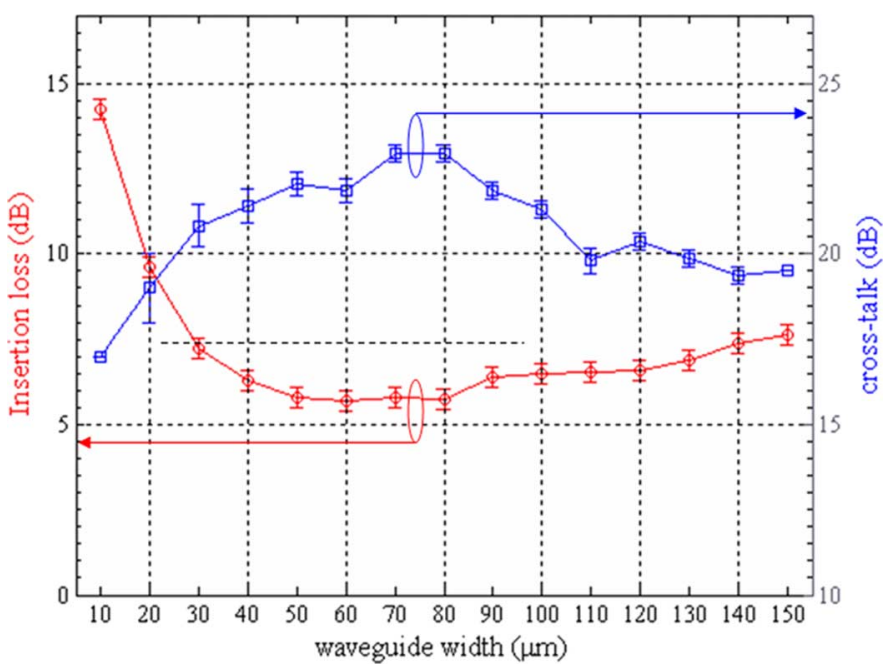

Fig. 6. Insertion loss and crosstalk measured on an array of 15, 50- $\mu \mathrm{m}$-thick waveguides with chirped width varying between $10-150 \mu \mathrm{m}$. For a given waveguide, the crosstalk shown corresponds to the mean crosstalk value due to one waveguide on its left and one waveguide on its right. The error bar is the difference between the crosstalk from the waveguide on the left and the crosstalk from the waveguide on the right.

the reference waveguide would experience from one waveguide on its left and one waveguide on its right in a real system. We then moved the PD and VCSEL to the next waveguide, repeated the same measurements and continued to measure all 15 waveguides in the array. The results, presented in Fig. 6 show that the coupling efficiency was optimum for waveguides with widths in the range between $50-80 \mu \mathrm{m}$ and that the lowest crosstalk SCR was obtained for waveguides having widths $70-80 \mu \mathrm{m}$. Therefore, the waveguides giving the maximum coupling efficiency combined with minimum crosstalk, SCR had widths in the range between $70-80 \mu \mathrm{m}$. So, we chose to use $70-\mu \mathrm{m}$ square waveguides in our prototype demonstrator.

In order to measure the insertion loss as a function of the three possible translational misalignments $x, y$, and $z$ we again used the same system of motorized stages with the modified MT-plug, as above. However, this time we scanned the optical input across the waveguides while monitoring the power at the pinhole, photodiode assembly which was fixed in position at the other end of one of the waveguides. The VCSEL bias current was set to $3 \mathrm{~mA}$, direct current, which provided an output optical power of $0 \mathrm{dBm}$. The position of maximum coupling efficiency (or equivalently minimum insertion loss) corresponded to $x=0, y=0, z=0$, where the insertion loss was measured to be $4.4 \mathrm{~dB}$. By scanning along $x$ and $y$ at different values of $z$ we generated the $x-y$ contour maps of insertion loss due to VCSEL misalignment [Fig. 7(a)]. The insertion loss shown in the contour maps is the difference relative to the minimum insertion loss. To investigate the effect of PD misalignments we exchanged the position of the PD and MT-plug on the motorized stages and repeated the experiments. We generated $x-y$ contour maps of the relative insertion loss due to PD misalignment [Fig. 7(b)]. The contour maps indicate the maximum misalignment that can be tolerated in the position of the VCSEL and the PD so that the insertion loss does not increase by more than a specified amount. Therefore, from these graphs we can
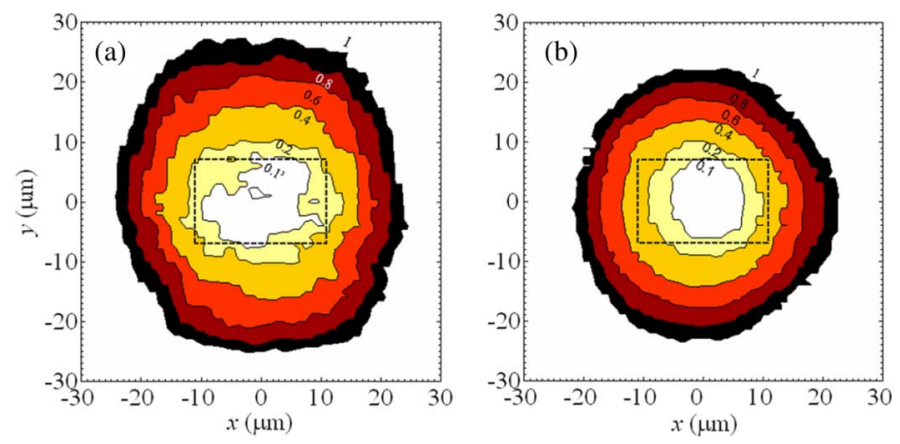

Fig. 7. (a) Contour map of relative insertion loss compared to the maximum coupling position for VCSEL misalignment at $z=0$. (b) Same for PD misalignment at $z=0$. Resolution step was $\Delta x=\Delta y=1 \mu \mathrm{m}$. Dashed rectangle in the middle of the maps corresponds to the expected relative insertion loss according to the calculated misalignments along $x$ and $y$ in Table I.

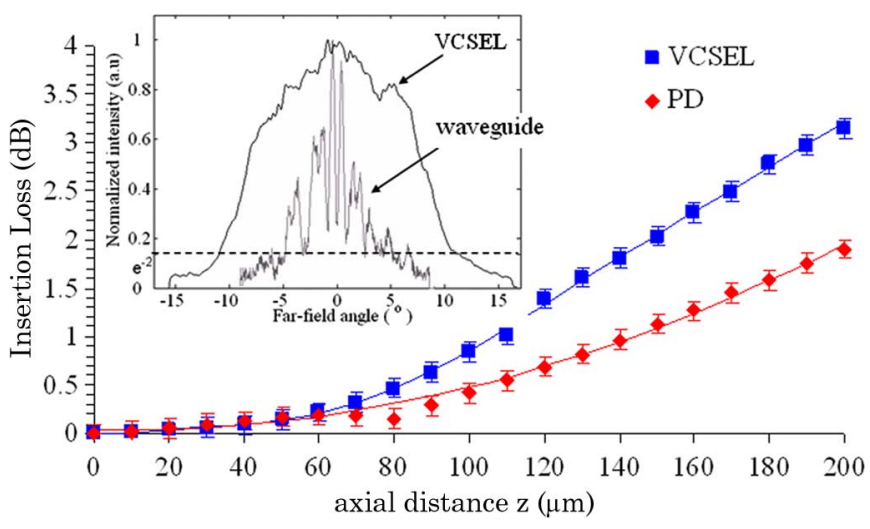

Fig. 8. Relative insertion loss of VCSEL and PD as they move away (along $z$ ) from the OPCB waveguides. Lateral position was set at the maximum coupling position, $x=y=0$. Inset: VCSEL and waveguide far-field angular divergence.

extract the tolerance requirements for a connector design. The dashed rectangles in the middle of the Fig. 7(a) and (b) indicate the maximum additional insertion loss due to the expected misalignments in Table I. As we see we expect a maximum of $0.2 \mathrm{~dB}$ additional loss due to VCSEL displacement and of 0.4 $\mathrm{dB}$ due to PD misalignment. Therefore, our connector should in total introduce a maximum of $0.6 \mathrm{~dB}$ of extra loss in the worst case of misalignment.

For axial misalignment measurements the MT-Plug, VCSEL, GRIN lens source assembly (or alternatively the pinhole, GRIN lens, PD detector assembly) was positioned at $x=y=0$ while at the other end the pinhole, GRIN lens, PD detector assembly (or alternatively the MT-Plug, VCSEL, GRIN lens source assembly) was butt-coupled to the waveguides. The power at the waveguide output was monitored as the source assembly (or alternatively the detector assembly) was moving axially covering the range $0<z<200 \mu \mathrm{m}$. From Fig. 8, we see that in both cases an axial separation of more than $80 \mu \mathrm{m}$ is required before the power drops by $0.1 \mathrm{~dB}$ and therefore this misalignment is not as critical as the lateral ones. At relatively large axial distances $z>80 \mu \mathrm{m}$ VCSEL axial misalignments cause greater losses than PD axial misalignments due to the larger angular divergence of the VCSEL field compared to the angular divergence of the field emerging from the waveguides. A horizontal cross section of the far field revealed an angular divergence (full angle 
at $\mathrm{e}^{-2}$ of maximum intensity) of $13^{\circ}$ for the waveguides well below the $23^{\circ}$ of the angular divergence of the VCSEL shown in the inset of Fig. 8.

\section{B. Loss Components}

At the best coupling position the insertion loss was 4.4-4.8 dB, without index matching fluid, depending on the choice of waveguide and experimental conditions, such as the way the substrate was mounted. When index-matching fluid, $n=1.557$ at $850 \mathrm{~nm}$, (similar to the core refractive index, $n_{\text {core }}=1.556$ ) was applied to the ends of the waveguides the insertion loss results improved by $2.1-2.7 \mathrm{~dB}$ which is the loss due to Fresnel reflections and scattering from the waveguide entrance and exit faces. The waveguide end faces were cut by a dicing saw but were not polished having a root mean square roughness of $\sim 38 \mathrm{~nm}$ and maximum size features of $\sim 200 \mathrm{~nm}$ [22]. Propagation loss due to polymer material absorption and scattering from the waveguide sidewalls was calculated with the aid of three 1-m spiral waveguides, fabricated under the same conditions, to be $0.08 \pm 0.01 \mathrm{~dB} / \mathrm{cm}$ giving a loss of $0.8 \mathrm{~dB}$ for our 10-cm-long waveguides. This loss is higher than the usually quoted figure of $0.03-0.04 \mathrm{~dB} / \mathrm{cm}$ for Truemode polymer [21]. This may be because the clean room facilities used to fabricate the waveguides had not been used for waveguide fabrication before and so had not been sufficiently characterized nor optimized. It may also be because the earlier figures were measured for $50-\mu \mathrm{m}$ square waveguides rather than for our $70-\mu \mathrm{m}$ square waveguides. The remaining $0.9-1.9 \mathrm{~dB}$ loss is the coupling loss between the VCSEL and waveguide and between the waveguide and the PD.

In published work on waveguide MT connectors, the lowest reported insertion loss of $3.5 \mathrm{~dB}$ was obtained using an actively aligned interposer in [5] but the authors did not report whether the waveguides used were polished. The authors of [6] reported a $1.6 \mathrm{~dB}$ insertion loss for their waveguide to waveguide connector but the waveguides were polished and active lateral alignment was used. A loss of $0.6 \mathrm{~dB}$ was reported in [14], for a multimode fiber to flexible film waveguide connector, with index-matching fluid and polished waveguides. Finally, an average insertion loss of $4.6 \mathrm{~dB}$ was reported in [15] for a fiber to polymer waveguide connector on a Si substrate. Our best insertion loss of $3.6 \pm 0.3 \mathrm{~dB}$ for two connections of $70-\mu \mathrm{m}$ square waveguides without index matching fluid, or $0.9 \pm 0.3 \mathrm{~dB}$ with index matching fluid is as good as the best achieved elsewhere to within experimental measurement accuracy. However, unlike the other published work these similar results have been achieved without the need for polishing of the waveguides and without any costly active alignment and so represent a major step forwards towards a commercially realizable low cost connector.

\section{Misaligned Crosstalk and SCR Discussion}

Misaligned components cause increased insertion loss and increased crosstalk which may substantially limit performance of high-bit rate systems. In order to examine crosstalk in our system the following experiment was performed. One VCSEL in the MT-plug was positioned at $y=0, z=0$ in front of a waveguide [denoted the 0th waveguide in Fig. 9(a)]. Then the
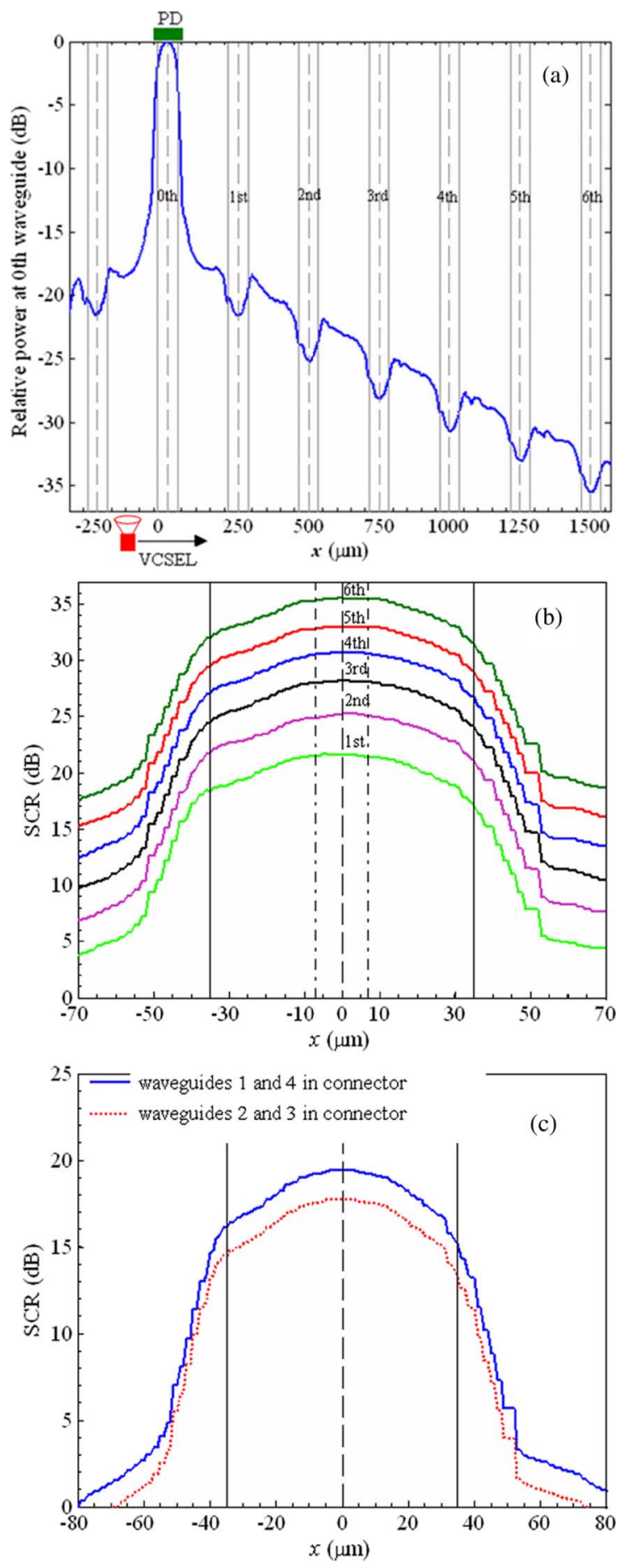

Fig. 9. (a) Power received at the end of 0th waveguide as a function of the lateral distance of the VCSEL from its center. The boundaries and the centers of the waveguides on the backplane are marked. In the cladding power drops at a rate of $0.011 \mathrm{~dB} / \mu \mathrm{m}$ (b) SCR levels that 0th waveguide experiences from its adjacent waveguides. (c) SCR experienced by waveguides number 1 and 4 and of waveguides number 2 and 3 from the array of four in the connector if all are in use. Dashed-dotted lines determine the boundaries of the maximum expected crosstalk based on current connector tolerances.

VCSEL was moved laterally in the range $-300 \mu \mathrm{m}<x<$ $+1600 \mu \mathrm{m}$ in $1-\mu \mathrm{m}$ steps while the power at the end of the 0th waveguide was monitored after each step. Since the waveguide lateral separation is $250 \mu \mathrm{m}$ this motion spanned the range between one waveguide to the left and six waveguides to the right 
of the 0th waveguide. While this VCSEL was scanning within the lateral edges of the 0 th waveguide the recorded power corresponded to the power coupled into this waveguide as a function of the lateral misalignment $x$. However, when the VCSEL had moved sufficiently that it was scanning the core region of the waveguide adjacent to the 0th waveguide, the power detected corresponded to crosstalk originating from other VCSELs in the same array that would be coupled to these waveguides in the multi-VCSEL plug assembly. Fig. 9(a) shows that as we move the VCSEL away from 0th waveguide so that light enters the cladding, the power drops almost linearly at a rate of $0.011 \mathrm{~dB} / \mu \mathrm{m}$, until a neighboring waveguide is reached where a narrow power reduction valley is observed due to power being coupled strongly into that waveguide core. The lowest crosstalk levels are achieved when perfect alignment occurs between the VCSELs and the waveguides. As VCSELs move away from the center of the other waveguides while still remaining in the core region the received power increased, indicating worsening of crosstalk. This measurement assumes that all VCSELs of the same array have the same amount and direction of misalignment simultaneously as would be the case in the MT plug assembly described. For a given lateral misalignment $\Delta x$ of the VCSEL from the center of a specific waveguide, we define the SCR to be the ratio of the signal power emerging from that specific waveguide to the crosstalk signal power emerging from the same waveguide when the VCSEL is misaligned from the center of the entrance of a neighboring waveguide by the same amount, $\Delta x$. This definition is useful since the VCSELs have a fixed spacing in the plug connector so that they all offset together by the same amount due to misalignment of the plug. Fig. 9(a) shows the SCR as a function of lateral misalignment of the source. The SCR measured at the 0th waveguide output when the VCSEL is misaligned so much that it is at the entrance of each of the six waveguides is shown in Fig. 9(b). In a real system crosstalk power from all neighboring waveguides combine in a specific waveguide. If we assume that the crosstalk signals do not interfere with the main signal, in other words that they are not coherently related which is reasonable since they arise from different lasers and the optical distance that they travel differs by more than their coherence lengths from the main signal then we can add their powers. This is shown in Fig. 9(c) as the combined SCR for an array of four VCSELs in the plug connector assembly. Since the waveguides have different optical environments, two curves are shown, one for waveguides number 1 and 4 which have three waveguides on one side of them, and one for waveguides number 2 and 3 which have one waveguide on one side and two waveguides on the other side. Clearly, the crosstalk is larger for waveguides numbered 2 and 3 as they have two close waveguides. For perfect alignment, the SCR is $17.76 \mathrm{~dB}$ for waveguides 2 and 3 and $19.46 \mathrm{~dB}$ for waveguide 1 and 4 . The SCR at the worst estimated misalignment is $17.43 \mathrm{~dB}$ for waveguides 2 and 3 and is $19.07 \mathrm{~dB}$ for waveguide 1 and 4 . Therefore, the maximum variation in SCR expected in our self-alignment technique is $0.33 \mathrm{~dB}$ for waveguides 2 and 3 or zero to within the experimental measurement accuracy and $0.39 \mathrm{~dB}$ for waveguides 1 and 4 . As waveguides 2 and 3 are the most important since they have the worst, SCR limiting performance the zero change in SCR for them means the alignment tolerances of the connector are sufficient.

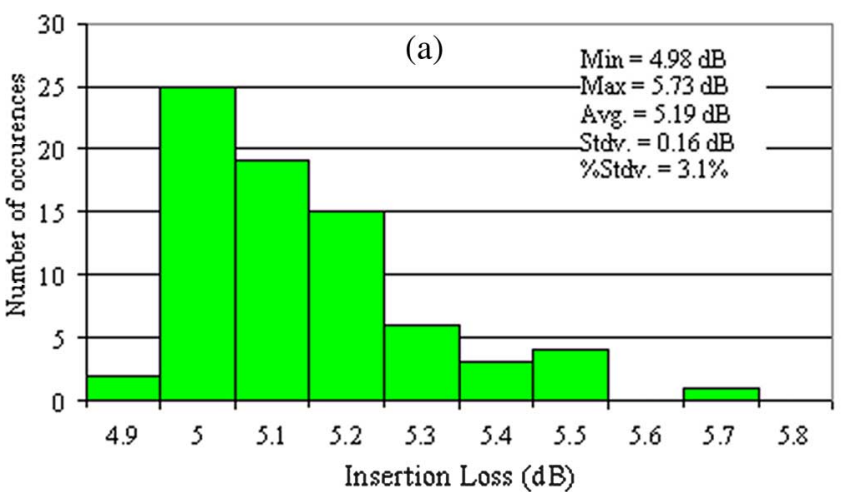

(b)

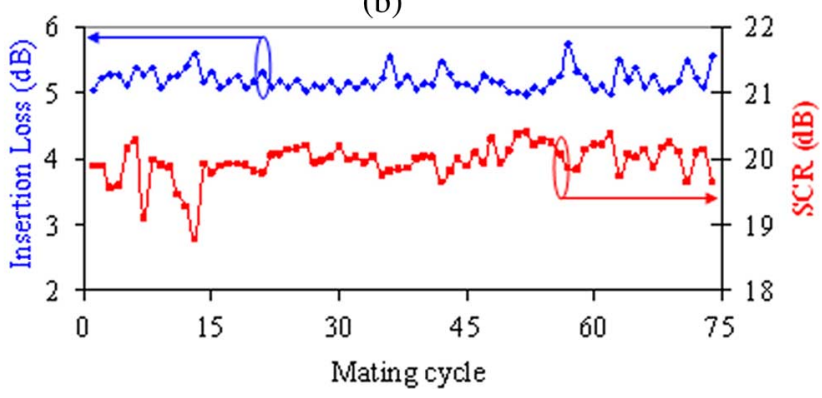

Fig. 10. (a) Insertion loss and SCR as a function of mating cycle for 75 engagements. (b) Histogram of insertion loss.

To the best of our knowledge, these are the first results of crosstalk reported as a function of source misalignment and the first time it has been appreciated that the position of the waveguides within the array has an effect on the worst crosstalk values.

\section{Repeatability}

We measured the reproducibility of insertion loss [Fig. 10(a)] and crosstalk for the best channel [Fig. 10(b)] for 75 engagements/disengagements of the MT-socket with the MT-plug. For the insertion loss measurements, only one VCSEL corresponding to waveguide number 1 was switched on and its output power level was set to $0 \mathrm{dBm}$ while the other three VCSELs of the array were switched off. In this way, only the power from the VCSEL of interest was recorded. The insertion loss was measured to be $5.19 \pm 0.16 \mathrm{~dB}$ for waveguides on this wafer, including propagation loss. The measurement accuracy of $\pm 0.16 \mathrm{~dB}$ was less than that earlier, $\pm 0.3 \mathrm{~dB}$ so the connector alignment reproducibility is better than that of our motorized stage measurement system. The histogram of insertion loss values obtained in Fig. 10(b) has the usual form of a one-sided Gaussian for a well-aligned connector. The most frequently encountered insertion loss was in the range of $5.1-5.2 \mathrm{~dB}$. The variation agrees well with that expected from the contour map values (Fig. 7) within the worst estimated misalignment. The standard deviation of insertion loss, 0.16 $\mathrm{dB}$, achieved by our alignment technique is better than the 0.34 $\mathrm{dB}$ reported in [24] and comparable to the $0.12 \mathrm{~dB}$ achieved in [25]. The literature [25] describes a single mode fiber to single mode fiber MT connector so the repeatability of our alignment technique is comparable with that achieved in single mode fiber MT connectors. 
In order to validate the assumption that the crosstalk from different VCSELs is not coherently related which allows us to add the powers of the individually measured crosstalk from each VCSEL we carried out a validation experiment. We switched the VCSEL of channel 1 off and turned on the other three VCSELs in the array with each set to a $0 \mathrm{dBm}$ output power. The combined crosstalk of the three VCSELs in neighboring channels was measured over 75 mating cycles [Fig. 10(a)] and the SCR found to be $19.95 \pm 0.27 \mathrm{~dB}$ which is in reasonable agreement with the highest value calculated and shown in Fig. 9(c) $19.46 \mathrm{~dB}$ given the experimental measurement accuracies, so validating the calculation technique.

\section{CONCLUSION}

A passive, precision, self-alignment technique is reported for coupling arrays of VCSELs and photodiodes to an array of buried channel waveguides which significantly reduces the cost of optical interconnections. A prototype multiple channel duplex connector using this approach was designed, constructed and tested.

The insertion loss for two connectors at best was $3.6 \mathrm{~dB}$ and could be reduced to at best $0.9 \mathrm{~dB}$ by using index-matching fluid. In a practical system there is, in addition, the potential to reduce the loss further by polishing, optimizing the dicing process and use of sufficient contact force.

The variation of optical coupling loss as a function of mechanical misalignments in $x, y$, and $z$ of both the laser source and of the photodetector were measured to assess how much misalignment may be permitted. Our self-alignment technique was estimated to have worst misalignment tolerances of in $x \pm$ $11 \mu \mathrm{m}$, in $y \pm 7 \mu \mathrm{m}$, and in $z+12.5 \mu \mathrm{m}$ which were shown to cause less than $0.2 \mathrm{~dB}$ additional insertion loss for the VCSEL and less than $0.4 \mathrm{~dB}$ additional insertion loss for the PD. However, in practice, the variation of insertion loss measured in multiple mating cycles was significantly less than this worst-case value being $0.16 \mathrm{~dB}$.

Insertion loss increases slowly with axial z VCSEL offsets to $80 \mu \mathrm{m}$, but increases at increasing rates beyond this. The loss due to $\mathrm{PD} z$ misalignments, however, does not increase so quickly with distance. PDs are less sensitive to $z$ but more sensitive to $x$ and $y$ misalignments due to the narrow $13^{\circ}$ divergent beam from the waveguide and their $75 \mu \mathrm{m}$ diameters in contrast to VCSELs due to their wider $23^{\circ}$ divergent beam and $7 \mu \mathrm{m} \mathrm{di-}$ ameters.

The SCR was found to decrease as the VCSEL was moved away from its perfectly aligned position. For perfect alignment, the SCR is $17.76 \mathrm{~dB}$ for waveguides in the center of the group and $19.46 \mathrm{~dB}$ for waveguide at the group edges. The SCR at the worst estimated misalignment is $17.43 \mathrm{~dB}$ for waveguides at the center of the group and is $19.07 \mathrm{~dB}$ for waveguides at the edges of the group. The loss of SCR, is negligible, as a result of estimated maximum misalignments for the waveguides at the center of the group which have the worst SCR. A very fast SCR drop off occurs for misalignments more than this and this is avoided by the used of the proposed alignment technique. Ideally, the crosstalk should be at least $10 \mathrm{~dB}$ better than these values. This could be achieved by an increased separation of the waveguides of $909 \mu \mathrm{m}$ resulting in a $1.159-\mathrm{mm}$ waveguide spacing which would limit the number of waveguide channels. In Fig. 10(b) it is noticeable that when the insertion loss improves the SCR also improves. Therefore, a better way to improve SCR is to reduce the coupling loss which raises the signal level. This could be achieved by index matching fluid, polishing and physical pressure. These are the first recorded measurements of crosstalk between adjacent and near neighbor waveguides when the connector is misaligned $\mathrm{d}$ which are crucial measurements as this is one of the main reasons this technology is being considered for replacing copper tracks.

During 75 repeated attaching and detaching cycles of the connector the insertion loss was measured to be $5.19 \pm 0.16 \mathrm{~dB}$ and the crosstalk was measured to be $-19.95 \pm 0.27 \mathrm{~dB}$ in reasonable agreement with the worst value $19.46 \mathrm{~dB}$ for the same waveguide obtained from misaligned insertion loss measurements, giving a small variation and good reproducibility. To the best of our knowledge, these are the first results for loss and loss histograms as a function of mating cycle for optical PCB MT connectors.

\section{ACKNOWLEDGMENT}

The authors would like to thank F. Tooley for helpful discussions and N. Suyal of Exxelis for supply of TruemodeTM polymer, fabrication, and dicing of the waveguides. The authors would also like to thank Dr. G. Yu for laying out the mask with the variable size waveguides and for carrying out initial experimental assessments.

\section{REFERENCES}

[1] E. Griese , "A high-performance hybrid electrical-optical interconnection technology for high-speed electronic systems," IEEE Trans. Adv. Packag., vol. 24, no. 3, pp. 375-383, Aug. 2001.

[2] R. T. Chen, L. Lin, C. C. Choi, Y. Liu, B. Bihari, L. Wu, R. Wickman, B. Picor, M. K. Hibbs-Brenner, J. Bristow, and Y. S. Liu, "Fully embedded board-level guided-wave optoelectronic interconnects," Proc. IEEE, vol. 88, no. 6, pp. 780-793, Jun. 2000.

[3] F. Mederer, R. Jäger, H. J. Unold, R. Michalzik, K. J. Ebeling, S. Lehmacher, A. Neyer, and E. Griese, "3-Gb/s data transmission with GaAs vcsels over PCB integrated polymer waveguides," IEEE Photon. Technol. Lett., vol. 13, pp. 1032-1034, Sep. 2001.

[4] J. Moisel, J. Guttman, H.-P. Huber, O. Krumpholz, M. Rode, R. Bogenberger, and K.-P. Kuhn, "Optical backplanes with integrated polymer waveguides,” Opt. Eng., vol. 39, no. 3, pp. 673-679, Mar. 2000.

[5] S. Hiramatsu and T. Mikawa, "Optical design of active interposer for high-speed chip level optical interconnects," J. Lightw. Technol., vol. 24, no. 2, pp. 927-934, Feb. 2006.

[6] S. Hiramatsu and M. Kinoshita, "Three-dimensional waveguide arrays for coupling between fiber-optic connectors and surface-mounted optoelectronic devices," J. Lightw. Technol., vol. 23, no. 9, pp. 2733-2739, Sep. 2005.

[7] G. Van Steenberge, P. Geerinck, S. Van Put, J. Van Koetsem, H. Ottevaere, D. Morlion, H. Thienpont, and P. Van Daele, "MT-compatible laser-ablated interconnections for optical printed circuit boards," J. Lightw. Technol., vol. 22, no. 9, pp. 2083-2090, Sep. 2004.

[8] C. Choi, L. Lin, Y. Liu, J. Choi, L. Wang, D. Haas, J. Magera, and R. T. Chen, "Flexible optical waveguide film fabrications and optoelectronic devices integration for fully embedded board-level optical interconnections," J. Lightw. Technol., vol. 22, no. 9, pp. 2168-2176, Sep. 2004.

[9] B. S. Rho, H. S. Cho, H.-H. Park, S.-W. Ha, and B.-H. (Tiger) Rhee, "PCB-compatible optical interconnection using $45^{\circ}$-ended connection rods and via-holed waveguides," J. Lightw. Technol., vol. 22, no. 9, pp. 2128-2134, Sep. 2004.

[10] T. Sakamoto, H. Tsuda, M. Hikita, T. Kagawa, K. Tateno, and C. Amano, "Optical interconnection using vcsels and polymeric waveguide circuits," J. Lightw. Technol., vol. 22, pp. 2083-2090, Sep. 2004.

[11] M. Hikita, R. Yoshimura, M. Usui, S. Tomaru, and S. Imamura, "Polymeric optical waveguides for optical interconnection," Thin Solid Film, vol. 331, pp. 303-308, , 1998. 
[12] K. Katsura, M. Usui, N. Sato, A. Ohki, N. Tanaka, N. Matsuura, T. Kagawa, K. Tateno, M. Hikita, R. Yoshimura, and Y. Ando, "Packaging for a 40-channel parallel optical interconnection module with an over-25 gbit/s throughput," IEEE Trans. Adv. Packag., vol. 22, no. 4, pp. 551-560, Nov. 1999.

[13] K. Byoung, I.-K. Cho, S. H. Ahn, M. Y. Jeong, D. J. Lee, Y. U. Heo, B. S. Rho, H.-H. Park, and B.-H. Rhee, "Optical backplane system using waveguide-embedded pcbs and optical slots," J. Lightw. Technol., vol. 22, no. 9, pp. 2119-2127, Sep. 2004.

[14] M. Hikita, S. Tomaru, K. Enbutsu, N. Ooba, R. Yoshimura, M. Usui, T. Yoshida, and S. Imamura, "Polymeric optical waveguide films for short-distance optical interconnects," IEEE J. Sel. Topics Quantum Electron., vol. 5, no. 5, pp. 1237-1242, Sep./Oct. 1999.

[15] H. Kosaka, M. Kajita, M. Yamada, and Y. Sugimoto, "A $16 \times 16$ optical full-cross-bar connection module with vcsel-array push/pull module and polymer-waveguide coupler connector," IEEE Photon. Technol. Lett., vol. 9, pp. 244-246, Feb. 1997.

[16] M. Schmatz, B. J. Offrein, and K. Vey, Optical interconnects: Intra-sSystem data transfer with light Apr. 25, 2005 [Online]. Available: http://www.zurich.ibm.com/pdf/sys/Optical_Interconnects@IBM-ZRL_2005-04_E.pdf

[17] Fiber Opt. Connector Intermateability Standard, Type MPO, TIA Standard 604-5.

[18] C. Fu, R. C. Brown, and C. Ume, "Temperature-dependent material characterizations for thin epoxy fr-4/e-glass woven laminate," in Proc. IEEE 43rd Electron. Compon. Technol. Conf. (ECTC '93), Orlando, FL, 1993, pp. 560-562.

[19] Military specification for plastic sheet, laminated, metal-clad for printed wiring board [Online]. Available: http://www.thetestlab.com/ data/13949_ss.pdf

[20] Navin Suyal, Exxelis Ltd., private communication, Jul. 2006.

[21] Coil Craft, Inc., Datasheets Tech. Rep., 2007 [Online]. Available: http://www.coilcraft.com

[22] A. Graham, "Novel optoelectronic devices for guided-wave and freespace optical interconnects," Ph.D. dissertation, School Eng. Physical Sci., Herriot-Watt Univ., Edinburgh, U.K., Apr. 2005.

[23] M. Takaya and K. Shibata, "Design and performance of very-high-density 60-fiber connectors," J. Lightw. Technol., vol. 21, pp. 1549-1556, Jun. 2003.

[24] T. S. Barry, D. L. Rode, M. H. Cordaro, R. R. Krchnavek, and K. Nakagawa, "Efficient multimode optical fiber-to-waveguide coupling for passive alignment applications in multichip modules," IEEE Trans. Compon. Packag. Manuf. Technol. B, vol. 18, no. 4, pp. 685-690, Nov. 1995.

[25] S. Nagasawa, Y. Yokoyama, F. Ashiya, and T. Satake, "A high-performance single-mode multifiber connector using oblique and direct endface contact between multipe fibers arranged in a plastic ferrule," IEEE Photon. Technol. Lett., vol. 3, pp. 937-939, Oct. 1991.

Ioannis Papakonstantinou (SM'03) was born in Athens, Greece. He received the diploma in electrical and computer engineering from National Technical University of Athens (NTUA), Greece, in 2000, and the M.Sc. degree in broadband technologies, under an Engineering and Physical Sciences Research Council (EPSRC) scholarship, which he was awarded with distinction, from the University College London (UCL), London, U.K., in 2002. He is currently working towards the Ph.D. degree in board-to-board high speed optical interconnections in the optical devices and system group at UCL, funded by Xyratex Ltd. and via an EPSRC doctoral training award.

He spent one year in the industry working on network management systems before moving to University College London.

Mr. Papakonstantinou is a student member of the Optical Society of America, (OSA). He received the Canon Foundation in Europe Research Fellowship in 2007. He served as a member of the program committee at the 18th and 19th
IEEE LEOS annual workshop on interconnections within high speed digital systems (HSD) and he chaired the Interconnect System Design session at the 18th HSD.

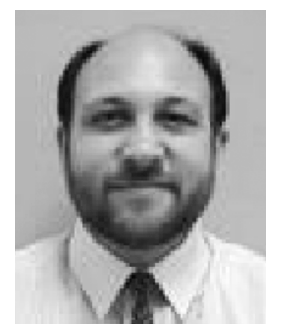

David R. Selviah (M'01) received the B.A. and M.A. degrees in physics and theoretical physics from Trinity College, Cambridge University, Cambridge, U.K., in 1980 and 1983, respectively.

He was with the Allen Clark Research Center, Plessey (Caswell), Ltd., Northamptonshire, U.K. (now Bookham Technology, Ltd.) for three years. He then joined the Department of Engineering Science, Oxford University, Oxford, U.K., where he was a Research Fellow for more than three years. In 1987, he joined the Department of Electronic and Electrical Engineering, University College London (UCL), London, U.K., where he is currently a Senior Lecturer. He has more than 100 publications and patents. His earlier research included airfield landing lights, aircraft streamlining, experimental particle physics, surface acoustic wave (SAW) adaptive correlator filters for RF phase-shift keyed (PSK) pattern recognition, PSK-to-minimum-shift keyed (MSK) conversion SAW filters and radar pulse compression SAW filters. His current research interests include $10 \mathrm{~Gb} / \mathrm{s}$ multimode polymer waveguide optical printed circuit boards with self aligning multichannel connectors, tuneable microlenses and filters, optical and computer generated holographic optical elements and storage, high sensitivity coherent detection, LCD backlights, higher order neural networks, pattern recognition and real time image processing. He is academic leader of the U.K. $£ 1.3$ million IeMRC Optical Printed Circuit Board Flagship Consortium of three universities and 10 companies.

Mr. Selviah is a member of the Institute of Physics, the Optical Society of America, and the European Optical Society.

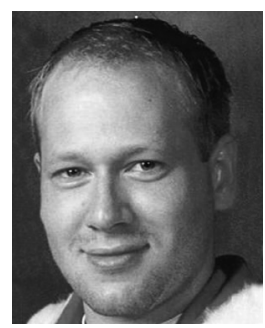

Richard C. A. Pitwon received the B.Sc. degree in physics (Hons.) from the University of St. Andrews, Fife, U.K., and the M.Sc. degree in computing science from Stirling University, Stirling, U.K., in 2001.

From 2002, he was enlisted by Xyratex Technology, Ltd, Havant, U.K., as part of the optical research and development team. During the past five years, he has focused on the development of enabling photonic technologies for optical printed circuit board systems, in particular high-speed pluggable optical backplane connector solutions. He has authored a number of white papers, conference paper,s and journal papers. $\mathrm{He}$ also holds 10 patents in the field of optical waveguide, PCB, and connector technologies.

Mr. Pitwon is currently a member of the steering committee of the (PAVT) Photonische Aufbau-und Verbindungs Technik group of the ITG (Informations Technische Gesellschaft).

Dave Milward received the B.Eng. degree in electrical and electronic engineering at Liverpool University, Liverpool, U.K.

Before joining Xyratex he spent 25 years working for IBM Hursley, initially as a digital design engineer before moving into management. Since 1994, he has worked as product development manager for Networking and Storage systems and more recently as project manager for collaborative research projects involving academic and industrial partners.

Mr. Milward is a Chartered Engineer and member of the Institution of Engineering and Technology (IET). 